\title{
First-principles study of competing ferroelectric and antiferroelectric instabilities in $\mathrm{BaTiO}_{3} / \mathrm{BaO}$ superlattices
}

\author{
Eric Bousquet, ${ }^{1,2}$ Javier Junquera, ${ }^{3}$ and Philippe Ghosez ${ }^{1}$ \\ ${ }^{1}$ Physique Théorique des Matériaux, Université de Liège, B-4000 Sart Tilman, Belgium \\ ${ }^{2}$ Materials Department, University of California, Santa Barbara, California 93106, USA \\ ${ }^{3}$ Departamento de Ciencias de la Tierra y Física de la Materia Condensada, Universidad de Cantabria, E-39005 Santander, Spain
}

(Received 22 April 2010; revised manuscript received 23 June 2010; published 23 July 2010)

\begin{abstract}
We report a first-principles study of $\left(\mathrm{BaTiO}_{3}\right)_{m} /(\mathrm{BaO})_{n}$ superlattices for a wide range of periodicities $m / n$. We show that such a system develops a polar zone-center instability for sufficiently large $m / n$ ratio, which can be understood, at least qualitatively, from a simple electrostatic model and should lead to a ferroelectric ground state. However, the analysis of the phonon-dispersion curves also points out the appearance of stronger antiferroelectric instabilities at the zone boundaries around $m=4$, before the critical ratio for ferroelectricity is reached and which still dominate beyond it. The dominant character of the antiferroelectric instability is explained from the depolarizing field which hardens the ferroelectric mode. This analysis allows us to predict that, $\left(\mathrm{BaTiO}_{3}\right)_{m} /(\mathrm{BaO})_{n}$ superlattices should present an antiferroelectric ground state for $m$ larger than 4 , which should smoothly evolve to a multidomain structure for increasing $m$ values and only become ferroelectric for large $m$.
\end{abstract}

DOI: 10.1103/PhysRevB.82.045426

PACS number(s): 77.80.Dj, 77.80.bn, 77.84.-s

\section{INTRODUCTION}

During the recent years, numerous works have been devoted to the study of interfacial effects in ferroelectric (FE) nanostructures. ${ }^{1,2}$ Both theoretical and experimental works have focused on various kinds of perovskite ferroelectric multilayers and superlattices including ferroelectric ultrathin films between metallic electrodes ${ }^{3-7}$ and superlattices combining either ferroelectric materials, ${ }^{8-10}$ ferroelectric and incipient-ferroelectric materials, ${ }^{11-21}$ or even incipientferroelectric and paraelectric materials. ${ }^{22,23}$ These studies highlighted the fact that three main factors govern the physics of multilayers: epitaxial strain, electrical boundary conditions, and interfacial effects.

The case of ferroelectric/insulator superlattices in which a ferroelectric perovskite alternates with a regular insulator having a nonperovskite structure was only marginally addressed in the literature. This might be due to the fact that no enhancement of the ferroelectric properties is expected in such systems due to the detrimental effect of the regular insulator on the ferroelectric properties. For some time, only the interface between $A \mathrm{O}$ and $A \mathrm{BO}_{3}$ oxides, where $A$ is an alkaline-earth atom such as $\mathrm{Sr}$ or $\mathrm{Ba}$, attracted some interest since $A O$ oxides are playing a key role as buffer layer in the epitaxial growth of perovskites directly on a silicon substrate. ${ }^{24} \mathrm{~A}$ first-principles study was previously reported by some of us for $\left(\mathrm{BaTiO}_{3}\right)_{m} /(\mathrm{BaO})_{n}$ and $\left(\mathrm{SrTiO}_{3}\right)_{m} /(\mathrm{SrO})_{n}$ superlattices grown on $\mathrm{Si},{ }^{25}$ but only the paraelectric periodicity $n=6$ and $m=5$ was considered and the ferroelectric properties were not explicitly discussed.

Nevertheless the focus on these particular FE/I interfaces has lately increased. The Ruddlesden-Poper series can be considered as an example of this type. ${ }^{26-28}$ This renewed interest is fueled by the possibility of modifying in a controlled way the delicate balance between all the instabilities present at the bulk $\mathrm{ABO}_{3}$ perovskites, that might be FE, antiferroelectric, and/or antiferrodistortive phase transitions by changing the composition and/or periodicity of the series. The perspectives of stabilizing new exotic phases not present at the bulk parent compounds is a strong driving force for these studies. ${ }^{14}$

In this paper, we study the case of $\left(\mathrm{BaTiO}_{3}\right)_{m} /(\mathrm{BaO})_{n}$ superlattices epitaxially grown on a $\mathrm{SrTiO}_{3}$ substrate as a prototypical example of FE/I superlattices. We first consider the predictions of a simple electrostatic model as reported in Ref. 13. Then, we present first-principles calculations of the phonon band structure of the paraelectric $\left(\mathrm{BaTiO}_{3}\right)_{m} /(\mathrm{BaO})_{n}$ superlattices for various layer thicknesses $m$ and $n$, and discuss the effects of the periodicity on the vibrational properties. We show that the predicted ground state differs from that of the simple electrostatic model and explain the reason for the discrepancy.

\section{TECHNICAL DETAILS}

First-principles simulations were performed in the framework of the density-functional theory (DFT) as implemented in the ABINIT package. ${ }^{29}$ All the results were calculated through the local-density approximation (LDA) for the exchange-correlation energy. An energy cutoff of 45 hartree was used for the plane-wave expansion. The Teter parametrization $^{30}$ for pseudopotentials was employed where the following orbitals were treated as valence states: $5 s, 5 p$, and $6 s$ for the Ba atom, $3 s, 3 p, 3 d$, and $4 s$ for the Ti atom, and $2 s$ and $2 p$ for the $\mathrm{O}$ atom. From the smallest to the biggest superlattices, tetragonal Monkhorst-Pack meshes from, respectively, $6 \times 6 \times 3$ to $6 \times 6 \times 1$ were considered for the Brillouin-zone sampling in order to produce accurate results. The vibrational properties and Born-effective charges were calculated using the density-functional perturbation theory. ${ }^{31}$

$\mathrm{BaTiO}_{3}$ (BTO) can be epitaxially grown on $\mathrm{BaO}$ (BO). ${ }^{32}$ The epitaxy is such that $A \mathrm{BO}_{3}(001) \| A \mathrm{O}(001)$ and 
$A \mathrm{BO}_{3}\langle 110\rangle \| A \mathrm{O}\langle 100\rangle$, which means that the $\mathrm{BaTiO}_{3}$ unit cell is rotated $45^{\circ}$ around the $(001) \mathrm{BaO}$ direction.

The superlattices were simulated by means of a supercell approximation with periodic boundary conditions, so shortcircuit electrostatic boundary conditions across the whole supercell are naturally imposed. To define the cell that is periodically repeated in space, a generic formula $\left(\mathrm{BaO}-\mathrm{TiO}_{2}\right)_{m} /(\mathrm{BaO})_{n}$ was used and labeled by $m / n$, where $n$ is the number of $\mathrm{BaO}$ oxide atomic layer and $m$ the number of $\mathrm{BaTiO}_{3}$ formula units. ${ }^{25,33}$

The epitaxial strain was treated by fixing the cubic inplane lattice constant of the superlattices. Only the case of an epitaxial strain corresponding to a hypothetical $\mathrm{SrTiO}_{3}$ substrate was considered (theoretical LDA relaxed cubic cell parameter of $\mathrm{SrTiO}_{3}: a=3.84 \AA$ ). For each thickness, we performed structural optimization of the atomic positions and the out-of-plane cell parameter of the superlattice in its highly symmetric phase corresponding to the tetragonal space group P4/ $\mathrm{mmm}$ (No. 123). To establish the notation, we will call the plane parallel to the interface the $(x, y)$ plane, whereas the perpendicular direction will be referred to as the $z$ axis.

\section{ELECTROSTATIC MODEL}

As explained in Refs. 1 and 13, the energy of a ferroelectric superlattice between materials 1 and 2 under short-circuit boundary conditions can be estimated within a simple model which assumes homogeneous polarization in each layer and neglect interface corrections, as the sum of the internal energy for the two compounds forming the superlattice and an electrostatic correction,

$$
E\left(P_{1}, P_{2}\right)=m U_{1}\left(P_{1}\right)+n U_{2}\left(P_{2}\right)+E_{\text {elec }}\left(m, n, P_{1}, P_{2}\right) .
$$

In first approximation, the internal energies $U_{1}$ and $U_{2}$ can be determined at the bulk level in the absence of a macroscopic electric field while the term $E_{\text {elec }}$ takes into account the electrostatic energy cost related to the polarization mismatch between the two types of layers. From now on, we will assume that material 1 is BTO and material 2 is BO.

\section{A. Bulk internal energies}

In their relaxed cubic structures, we obtained the lattice constants $a_{\mathrm{BO}}=5.46 \AA$ and $a_{\mathrm{BTO}}=3.94 \AA$ for $\mathrm{BaO}$ and $\mathrm{BaTiO}_{3}$, respectively, which are in good agreement with previous LDA results. ${ }^{25}$ In the $\mathrm{NaCl}$ structure, a cell parameter of $5.46 \AA$ in the $\mathrm{BaO}$ bulk corresponds to a $\mathrm{Ba}$-Ba distance of $3.86 \AA$. Therefore, when grown on a $\mathrm{SrTiO}_{3}$ substrate, assuming a theoretical cubic in-plane lattice constant of $3.84 \AA \mathrm{BaTiO}_{3}$ is under compressive strain and becomes tetragonal, as well as $\mathrm{BaO}$ (the epitaxial strain being applied on the Ba-Ba distance) in a smaller extent. ${ }^{34}$ After relaxation with this epitaxial constraint, the out-of-plane cell parameters of the paraelectric references are $c_{\mathrm{BO}}^{0}=5.48 \AA$ and $c_{\mathrm{BTO}}^{0}=4.01 \AA$ for $\mathrm{BaO}$ and $\mathrm{BaTiO}_{3}$, respectively (where the superscript " 0 " refers to the fact that it is the $c$ parameter at zero polarization). The associated Born-effective charge and electronic dielectric tensors are reported in Table I. These
TABLE I. Cell parameters ( $a$, in $\AA$ ), diagonal components of the Born-effective charge tensors $\left(Z^{*}\right.$, in $e$ ), diagonal components of the optical dielectric constant tensors $\left(\epsilon^{\infty}\right)$, frequency of the lowest TO modes $\left(\omega_{\mathrm{TO}}\right.$, in $\left.\mathrm{cm}^{-1}\right)$, and associated mode effective charges $\left(\bar{Z}^{*}\right.$, in $e$ ) for the bulk paraelectric $\mathrm{BaO}$ and $\mathrm{BaTiO}_{3}$, under the constraint of an in-plane lattice constant imposed by an hypothetical $\mathrm{SrTiO}_{3}$ substrate $(3.84 \AA) . \mathrm{O}_{1}$ refers to the $\mathrm{O}$ in the $\mathrm{BaO}$ plane while $\mathrm{O}_{2 / 3}$ stands for the two equivalent oxygen in the $\mathrm{TiO}_{2}$ planes. The diagonal components of $Z^{\star}$ and $\epsilon^{\infty}$ along $x$ and $y$ are equal by symmetry.

\begin{tabular}{lccccc}
\hline \hline & \multicolumn{2}{c}{$\mathrm{BaO}$} & & \multicolumn{2}{c}{$\mathrm{BaTiO}_{3}$} \\
\cline { 2 - 3 } \cline { 5 - 6 } & $x / y$ & $z$ & & $x / y$ & $z$ \\
\hline$a$ & 3.84 & 5.48 & & 3.84 & 4.01 \\
$Z^{*}(\mathrm{Ba})$ & 2.83 & 2.78 & & 2.81 & 2.77 \\
$Z^{*}\left(\mathrm{O}_{1}\right)$ & -2.83 & -2.78 & & -2.27 & -5.29 \\
$Z^{*}(\mathrm{Ti})$ & & & & 7.48 & 6.80 \\
$Z^{*}\left(\mathrm{O}_{2 / 3}\right)$ & & & & $-2.13 /-5.90$ & -2.14 \\
$\epsilon^{\infty}$ & 4.39 & 4.38 & & 6.65 & 6.45 \\
$\omega_{\mathrm{TO}}$ & 118 & 74 & & 195 & $180 i$ \\
$\bar{Z}^{*}$ & 3.14 & 3.08 & 8.00 & 8.47 \\
\hline \hline
\end{tabular}

values are comparable to those in the cubic structure with a small anisotropy due to the tetragonal symmetry: most components of the Born-effective charges and electronic dielectric tensors are smaller in the $z$ than in the $x / y$ directions. The frequency of the lowest transverse-optic (TO) mode is also reported in Table I together with their mode effective charges. In this constrained paraelectric configuration, $\mathrm{BaO}$ is stable but the mode polarized along the $z$ direction has a slightly lower frequency than those along the $x / y$ directions. ${ }^{35} \mathrm{BaTiO}_{3}$ shows a ferroelectric instability along the $z$ direction with an imaginary frequency of $180 i \mathrm{~cm}^{-1}$ and an associated mode effective charge of $8.47 e$ while in the $x / y$ directions, the frequency of the soft mode is shifted to real frequency $\left(195 \mathrm{~cm}^{-1}\right)$ and its mode effective charge is slightly reduced $(8.00 e)$.

The compressive epitaxial strain imposed by the substrate favors a ferroelectric $c$ phase ${ }^{36}$ and, therefore, $\mathrm{BaTiO}_{3}$ effectively becomes a uniaxial ferroelectric. In what follows, we shall assume that all the polarization and electric fields are directed along $z$. A full relaxation of both the atomic coordinates and lattice vectors gives rise to a ferroelectric ground state with a spontaneous polarization of $38 \mu \mathrm{C} / \mathrm{cm}^{2}$ and a relaxed out-of-plane cell parameter of $4.07 \AA$. The difference of energy between the ferroelectric ground state and the paraelectric reference is $\Delta E=16 \mathrm{meV}$, which corresponds to the depth of the double well associated to the evolution of the potential energy with the polarization (see red curve with squares in Fig. 1). In principle, the determination of the shape of the double-well potential energy, $U(P)$, would require calculation at constrained $P$ as proposed in Ref. 37 . Here we used a more approximate but still reasonable approach. First, we froze in different fractions of the pattern of atomic displacements $\xi_{\text {BTO }}$, defined as the difference between the atomic positions in the relaxed ground states and the paraelectric reference structure. Second, for each frozen fraction of $\xi_{\mathrm{BTO}}$, the $c$ cell parameter was relaxed in order to 


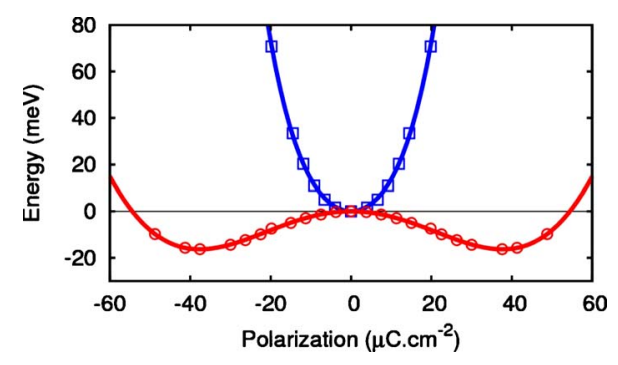

FIG. 1. (Color online) Energy as a function of spontaneous polarization for epitaxial bulks. The blue curve with squares corresponds to the tetragonal bulk $\mathrm{BaO}$ and the red curve with circles is the double well of tetragonal $\mathrm{BaTiO}_{3}$ bulk. For each single-point energy, the out-of-plane cell parameter was relaxed.

include the strain effects in the internal energy. Then, the corresponding polarization was approximated by $P_{\mathrm{BTO}}^{0}$ $=\frac{\Sigma_{i} \xi_{\mathrm{BTO}, i} Z_{i}^{*}}{\Omega}$, where $\xi_{\mathrm{BTO}, i}$ is the displacement of atom $i$ with respect to its high symmetric position, $Z_{i}^{*}$ its Born-effective charge [with the geometry used in the present work, the $(z, z)$ component of the Born-effective charge tensor], and $\Omega$ the volume of the unit cell. The 0 in the superscript makes reference to the fact that this polarization is estimated assuming zero macroscopic electric field. Finally, the internal energy is plotted as a function of the zero-field polarization (red curve in Fig. 1) and fitted to a polynomial expansion,

$$
U_{\mathrm{BTO}}\left(P_{\mathrm{BTO}}^{0}\right)=A_{\mathrm{BTO}} P_{\mathrm{BTO}}^{0^{2}}+B_{\mathrm{BTO}} P_{\mathrm{BTO}}^{0^{4}}+C_{\mathrm{BTO}} P_{\mathrm{BTO}}^{0^{6}}
$$

where $A_{\mathrm{BTO}}, B_{\mathrm{BTO}}$, and $C_{\mathrm{BTO}}$ are fitting parameters, reported in Table II. The sixth-order term was needed to ensure better agreement of the fit with the first-principles data.

The only way to polarize $\mathrm{BaO}$ is within the subspace spanned by its $\mathrm{TO}$ modes. In the superlattice, the $\mathrm{BaO}$ layer is expected to be polarized by $\mathrm{BaTiO}_{3}$ along the $z$ direction and so the evolution of its associated internal energy $U_{\mathrm{BO}}$ was determined by freezing the pattern of displacements of the TO mode along the $z$ direction given in Table I. As for $\mathrm{BaTiO}_{3}$ the out-of-plane cell parameter was relaxed for each amplitude of the frozen pattern of displacements. Since $\mathrm{BaO}$ is not ferroelectric, to polarize it has an energy cost and the shape of the internal energy corresponds to a single well (blue curve in Fig. 1) that can be approximated as

$$
U_{\mathrm{BO}}\left(P_{\mathrm{BO}}^{0}\right)=A_{\mathrm{BO}} P_{\mathrm{BO}}^{0^{2}}+B_{\mathrm{BO}} P_{\mathrm{BO}}^{0^{4}},
$$

where $A_{\mathrm{BO}}$ and $B_{\mathrm{BO}}$ are also fitting parameters reported in Table II. Here again, a fourth-order term has been included to achieve a better agreement in the fit of the energy which

TABLE II. Values of the parameters, used in Eqs. (2) and (3), that result from a fit of the bulk internal energies under zero macroscopic field versus zero-field polarization shown in Fig. 1. Units required to produce energies in millielectron volt when the polarization enters in microcoulomb per centimeter square.

\begin{tabular}{ccccc}
\hline \hline$A_{\text {Вто }}$ & $B_{\text {ВТО }}$ & $C_{\text {ВTO }}$ & $A_{\text {ВО }}$ & $B_{\text {ВО }}$ \\
\hline-0.0239 & $9.369 \times 10^{-6}$ & $-4.373 \times 10^{-10}$ & 0.122 & $1.514 \times 10^{-4}$ \\
\hline \hline
\end{tabular}

slightly deviates from the harmonic approximation at larger amplitude of polarizations.

\section{B. Electrostatic energy cost}

In ferroelectric/incipient-ferroelectric superlattices, the incipient-ferroelectric layer is expected to become polarized with nearly the same spontaneous polarization as the ferroelectric layer, resulting in a roughly homogeneous polarization through the whole structure, ${ }^{13}$ so that $E_{\text {elec }}$ in Eq. (1) will vanish. Here, in the $\mathrm{BaTiO}_{3} / \mathrm{BaO}$ superlattices, the $\mathrm{BaO}$ layer is less polarizable and is no more expected to have necessarily the same polarization than the $\mathrm{BaTiO}_{3}$ layer. The polarization misfit gives rise to electric fields and produce an additional electrostatic energy cost. Assuming that both layers are homogeneously polarized, its expression can be deduced from the expansion of the total energy of a dielectric with respect to the electric field $\mathcal{E} \cdot{ }^{37}$ For the case of a single dielectric,

$$
E(\mathcal{E})=E(\mathcal{E}=0)-\mathcal{E} P^{0}-\frac{1}{2} \epsilon_{0} \epsilon^{\infty} \mathcal{E}^{2}
$$

Generalizing Eq. (4) to the present context, where we have two dielectrics periodically repeated in the superlattice, then the zero-field energy can be written as

$$
E(\mathcal{E}=0)=m U_{\mathrm{BTO}}\left(P_{\mathrm{BTO}}^{0}\right)+n U_{\mathrm{BO}}\left(P_{\mathrm{BO}}^{0}\right)
$$

and the electrostatic energy for the superlattices can be written as

$$
\begin{aligned}
E_{\text {elec }}( & \left.P_{\mathrm{BTO}}^{0}, P_{\mathrm{BO}}^{0}, \mathcal{E}_{\mathrm{BTO}}, \mathcal{E}_{\mathrm{BO}}\right) \\
= & -\Omega_{\mathrm{BTO}} \mathcal{E}_{\mathrm{BTO}} P_{\mathrm{BTO}}^{0}-\frac{\Omega_{\mathrm{BTO}}}{2} \epsilon_{0} \epsilon_{\mathrm{BTO}}^{\infty} \mathcal{E}_{\mathrm{BTO}}^{2} \\
& -\Omega_{\mathrm{BO}} \mathcal{E}_{\mathrm{BO}} P_{\mathrm{BO}}^{0}-\frac{\Omega_{\mathrm{BO}}}{2} \epsilon_{0} \epsilon_{\mathrm{BO}}^{\infty} \mathcal{E}_{\mathrm{BO}}^{2},
\end{aligned}
$$

where $\Omega_{\mathrm{BTO}}$ and $\Omega_{\mathrm{BO}}$ are, respectively, the volumes of the $\mathrm{BaTiO}_{3}$ and the $\mathrm{BaO}$ layers and can be estimated as

$$
\begin{gathered}
\Omega_{\mathrm{BTO}}=m c_{\mathrm{BTO}} S, \\
\Omega_{\mathrm{BO}}=n c_{\mathrm{BO}} S
\end{gathered}
$$

with $S$ the area of the surface of the unit cell $\left[S=\left(\begin{array}{ll}3.84 & \AA\end{array}\right)^{2}\right]$ and $c_{\mathrm{BTO}}$ and $c_{\mathrm{BO}}$ are approximated by $c_{\mathrm{BTO}}^{0}$ and $c_{\mathrm{BO}}^{0} \cdot{ }^{38}$

The electric fields inside the $\mathrm{BaTiO}_{3}$ layer, $\mathcal{E}_{\text {BTO}}$, and the $\mathrm{BaO}$ layer, $\mathcal{E}_{\mathrm{BO}}$, are not independent. From the short-circuit boundary conditions across the whole supercell,

$$
m c_{\mathrm{BTO}} \mathcal{E}_{\mathrm{BTO}}+n c_{\mathrm{BO}} \mathcal{E}_{\mathrm{BO}}=0,
$$

the continuity of the normal component of the displacement field at the interface,

$$
\epsilon_{0} \mathcal{E}_{\mathrm{BTO}}+P_{\mathrm{BTO}}=\epsilon_{0} \mathcal{E}_{\mathrm{BO}}+P_{\mathrm{BO}}
$$

and the constitutive relations 
TABLE III. Polarizations $P_{\mathrm{BTO}}^{0}$ and $P_{\mathrm{BO}}^{0}\left(\mu \mathrm{C} / \mathrm{cm}^{2}\right)$ minimizing the total energy of Eq. (14) for a range of different layer thicknesses $n$ and $m$. The corresponding internal, electrostatic, and total energies (respectively, $U, E_{\text {elec }}$, and $\left.E_{\text {tot }}\right)$ are also reported. Units of the energies in millielectron volt.

\begin{tabular}{rrrrrrr}
\hline \hline$n$ & $m$ & $P_{\mathrm{BTO}}^{0}$ & $P_{\mathrm{BO}}^{0}$ & \multicolumn{1}{c}{$U$} & $E_{\text {elec }}$ & $E_{\text {tot }}$ \\
\hline 2 & 7 & 3.2 & 2.2 & -0.58 & 0.57 & -0.01 \\
2 & 8 & 9.6 & 6.3 & -6.69 & 5.55 & -1.14 \\
2 & 9 & 13.0 & 8.4 & -14.95 & 11.03 & -3.92 \\
2 & 10 & 15.4 & 9.9 & -24.77 & 16.64 & -8.13 \\
2 & 11 & 17.3 & 11.0 & -35.76 & 22.17 & -13.59 \\
4 & 14 & 3.2 & 2.2 & -1.17 & 1.14 & -0.03 \\
4 & 15 & 7.2 & 4.8 & -6.74 & 6.03 & -0.71 \\
4 & 16 & 9.6 & 6.3 & -13.38 & 11.10 & -2.28 \\
4 & 17 & 11.4 & 7.5 & -21.10 & 16.43 & -4.67 \\
4 & 18 & 13.0 & 8.4 & -29.90 & 22.07 & -7.83 \\
6 & 21 & 3.2 & 2.2 & -1.75 & 1.71 & -0.04 \\
6 & 22 & 6.2 & 4.1 & -7.09 & 6.52 & -0.58 \\
6 & 23 & 8.1 & 5.4 & -13.19 & 11.48 & -1.71 \\
6 & 24 & 9.6 & 6.3 & -20.07 & 16.65 & -3.42 \\
6 & 25 & 10.9 & 7.1 & -27.79 & 22.11 & -5.68 \\
\hline \hline
\end{tabular}

$$
\begin{gathered}
P_{\mathrm{BTO}}=P_{\mathrm{BTO}}^{0}+\epsilon_{0} \chi_{\mathrm{BTO}}^{\infty} \mathcal{E}_{\mathrm{BTO}}, \\
P_{\mathrm{BO}}=P_{\mathrm{BO}}^{0}+\epsilon_{0} \chi_{\mathrm{BO}}^{\infty} \mathcal{E}_{\mathrm{BO}},
\end{gathered}
$$

we can arrive to expressions for $\mathcal{E}_{\mathrm{BTO}}$ and $\mathcal{E}_{\mathrm{BO}}$,

$$
\begin{gathered}
\mathcal{E}_{\mathrm{BTO}}=-\frac{n c_{\mathrm{BO}}\left(P_{\mathrm{BTO}}^{0}-P_{\mathrm{BO}}^{0}\right)}{\epsilon_{0}\left(n c_{\mathrm{BO}} \epsilon_{\mathrm{BTO}}^{\infty}+m c_{\mathrm{BTO}} \epsilon_{\mathrm{BO}}^{\infty}\right)}, \\
\mathcal{E}_{\mathrm{BO}}=\frac{m c_{\mathrm{BTO}}\left(P_{\mathrm{BTO}}^{0}-P_{\mathrm{BO}}^{0}\right)}{\epsilon_{0}\left(n c_{\mathrm{BO}} \epsilon_{\mathrm{BTO}}^{\infty}+m c_{\mathrm{BTO}} \epsilon_{\mathrm{BO}}^{\infty}\right)} .
\end{gathered}
$$

Replacing Eqs. (11a) and (11b) into Eq. (6), then the electrostatic energy reduces to

$$
E_{\text {elec }}\left(n, m, P_{\mathrm{BTO}}^{0}, P_{\mathrm{BO}}^{0}\right)=C(n, m)\left(P_{\mathrm{BTO}}^{0}-P_{\mathrm{BO}}^{0}\right)^{2},
$$

where

$$
C(n, m)=\frac{S}{2 \epsilon_{0}} \frac{n m c_{\mathrm{BO}} c_{\mathrm{BTO}}}{\left(m c_{\mathrm{BTO}} \epsilon_{\mathrm{BO}}^{\infty}+n c_{\mathrm{BO}} \epsilon_{\mathrm{BTO}}^{\infty}\right)} .
$$

Here again, we will approximate $c_{\mathrm{BTO}}$ and $c_{\mathrm{BO}}$ by $c_{\mathrm{BTO}}^{0}$ and $c_{\mathrm{BO}}^{0}$.

Combining Eqs. (5) and (12), the total energy for the electrostatic model for $\left(\mathrm{BaTiO}_{3}\right)_{m} /(\mathrm{BaO})_{n}$ superlattices reads

$$
\begin{aligned}
E\left(n, m, P_{\mathrm{BTO}}^{0}, P_{\mathrm{BO}}^{0}\right)= & m U_{\mathrm{BTO}}\left(P_{\mathrm{BTO}}^{0}\right)+n U_{\mathrm{BO}}\left(P_{\mathrm{BO}}^{0}\right) \\
& +C(n, m)\left(P_{\mathrm{BTO}}^{0}-P_{\mathrm{BO}}^{0}\right)^{2} .
\end{aligned}
$$

\section{Minimization of the total energy}

For each periodicity $m / n$, the ground state of the superlattice can be estimated by minimizing the total energy, Eq. (14), with respect to $P_{\mathrm{BTO}}^{0}$ and $P_{\mathrm{BO}}^{0}$. Then, from the knowl- edge of the zero-field polarization, the constitutive relations [Eqs. (10a) and (10b)], and the electric fields [Eqs. (11a) and (11b)], we can infer values for the total polarization inside each layer. In Table III we report the results of such minimization for a set of periodicities $n / m$. It is interesting to see that at a fixed number of $\mathrm{BaO}$ layers $n$, the model predicts a critical ratio $m / n=3.5$ beyond which the system becomes ferroelectric: $7 / 2,14 / 4$, and $21 / 6$. For all of these ferroelectric states, the minimum of energy is reached when the polarization of the $\mathrm{BaO}$ layer is about $35 \%$ smaller than the polarization of the $\mathrm{BaTiO}_{3}$ layer, giving rise to an electrostatic energy of the same order of magnitude than the internal energy. We notice that, considering the internal energy alone (i.e., assuming $P_{\mathrm{BTO}}^{0}=P_{\mathrm{BO}}^{0}$ and $\left.E_{\text {elec }}=0\right)$, we can also predict a critical ratio $m / n \simeq 5$ to get a ferroelectric state $(11 / 2,21 / 4$, and 31/6). This critical ratio coming from the pure internal energies is however larger than the critical ratio $(\mathrm{m} / \mathrm{n}$ $\simeq 3.5$ ) calculated with the electrostatic energy, meaning that the quantity of $\mathrm{BaTiO}_{3}$ to get ferroelectricity in the superlattice is lowered by polarizing the $\mathrm{BaO}$ layer less than the $\mathrm{BaTiO}_{3}$ layer, a situation distinct from that usually observed in ferroelectric/incipient-ferroelectric superlattices. As the polarizability of the insulating materials increases however, we will tend toward the situation where $P_{\mathrm{BTO}}^{0}=P_{\mathrm{BO}}^{0}$.

\section{FIRST-PRINCIPLES RESULTS}

As a second step, we have performed DFT calculations (i) to verify to which extent the predictions of the simple electrostatic model are verified and (ii) to analyze the eventual existence of instabilities in the phonon spectra that would indicate the existence of structural phase transitions.

\section{A. Paraelectric reference}

In its paraelectric reference state with space group $P 4 / \mathrm{mmm}$ (No. 123), the in-plane atomic positions in each 
TABLE IV. Out-of-plane cell parameter, $c^{0}$, for a range of different layer thicknesses $n$ and $m . c_{\text {relax }}^{0}$ represents the perpendicular cell parameter of the paraelectric relaxed case, $c_{\text {bulk }}^{0}$ stands for the sum of the corresponding bulk unit cell, $c_{\text {bulk }}^{0}=m c_{\mathrm{BTO}}^{0}+\frac{n}{2} c_{\mathrm{BO}}^{0}$. The last column reports the deviation between $c_{\text {bulk }}^{0}$ and $c_{\text {relax }}^{0}$. Units in angstrom.

\begin{tabular}{lrrrr}
\hline \hline$n$ & $m$ & $c_{\text {relax }}^{0}$ & $c_{\text {bulk }}^{0}$ & Deviation \\
\hline 2 & 2 & 13.43 & 13.50 & -0.07 \\
2 & 4 & 21.45 & 21.52 & -0.07 \\
2 & 6 & 29.46 & 29.54 & -0.08 \\
2 & 8 & 37.48 & 37.56 & -0.08 \\
4 & 2 & 18.91 & 18.98 & -0.07 \\
4 & 4 & 26.93 & 27.00 & -0.07 \\
4 & 6 & 34.95 & 35.02 & -0.07 \\
4 & 8 & 42.95 & 43.04 & -0.09 \\
4 & 10 & 50.99 & 51.06 & -0.07 \\
\hline
\end{tabular}

layer correspond to the bulk high symmetric positions. In the out-of-plane direction a mirror-symmetry plane is present at the center of each layer. Since in the $z$ direction the atoms are not in their high symmetry positions, atomic relaxations take place and give rise to rumpling distortions. We recover the previous first-principles results of Ref. 25, showing that the rumpling distortions are mainly localized at the interface between the layers and rapidly vanish at the center of thick enough layers, in good agreement also with the "locality principle" discussed in Ref. 7. This interface effect in the superlattices involves also a modification of the interplanar distances between atomic planes with respect to those in the bulks but this effect is also strongly located at the interface between the two layers. ${ }^{25}$ As shown in Table IV, this feature tends to compress the total out-of-plane cell parameter of the multilayer. In spite of the different periodicities considered, a constant deviation is calculated between the bulk references and the relaxed superlattices confirming the fact that the differences with respect to the bulks are mainly located at the interface between the two layers.

Since the ferroelectric instabilities are strongly sensitive to the volume and pressure, the reduction in the out-of-plane thicknesses in the superlattices could strongly influence the out-of-plane ferroelectric soft mode for the smallest periodicities. For example, in the $2 / 2$ periodicity, this reduction with respect to the bulk reference equals $0.07 \AA$, which corresponds to an equivalent pressure of about $1 \mathrm{GPa}$.

\section{B. Zone-center instability}

For two fixed $\mathrm{BaO}$ thicknesses, $n=2$ and $n=4$, the evolution of the square of the frequency of the lowest zone-center TO mode along the out-of-plane direction $\left(\mathrm{FE}_{z}\right), \omega_{\mathrm{TO}}^{2}$, calculated in the relaxed paraelectric references, is shown in Fig. 2. This $\mathrm{FE}_{z}$ mode, that after condensation will be responsible of the ferroelectric transition, is strongly sensitive to the thickness of $\mathrm{BaTiO}_{3}$ layer, as expected since the ferroelectric instability is coming from $\mathrm{BaTiO}_{3}$. As shown in Fig. 2, $\omega_{\text {TO }}^{2}$ goes from high values at the smallest $m$ and decreases lin-

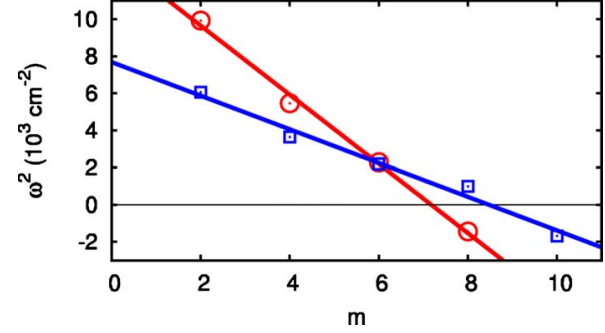

FIG. 2. (Color online) Square of the frequency of the lowest zone-center out-of-plane transverse optic mode, $\omega_{\mathrm{TO}}^{2}$, for $n=2$ (red, circles) and $n=4$ (blue, squares) as a function of the $\mathrm{BaTiO}_{3}$ thickness, $m$. The phonon frequencies have been computed in the reference paraelectric structure.

early with the increase in the number of $\mathrm{BaTiO}_{3}$ unit cells for both $n=2$ and $n=4 \mathrm{BaO}$ thicknesses. Beyond a critical $m / n$ periodicity, 7/2 and $9 / 4$, the $\mathrm{FE}_{z}$ mode becomes unstable (negative $\omega_{\mathrm{TO}}^{2}$ ). This means that, in spite of the nonferroelectric character of $\mathrm{BaO}$ layer, the entire supercell can develop a ferroelectric instability when a critical ratio between $m / n$ is reached.

This confirms the results predicted with the simple electrostatic model, Eq. (14). The agreement is amazingly good for $n=2$ but the critical periodicities at which the ferroelectricity appears do not correspond to a constant ratio as inferred from the model: $7 / 2$ and 9/4 from the first-principles phonon calculations instead of $7 / 2$ and $14 / 4$ from the electrostatic model. Since the electrostatic model is built through the bulk soft-mode eigendisplacements and the bulk Borneffective charges (to estimate the polarization), we now investigate to which extent this constitutes a reasonable approximation.

To highlight the differences between the bulks and the superlattice we report in Table $\mathrm{V}$ the Born-effective charges of individual atoms at the interface and in the middle of each layer of the superlattice in the paraelectric reference structure. In the middle of the $\mathrm{BaO}$ layer, the amplitude of the $z z$ component of the Born-effective charges are larger than their bulk values, a difference that is amplified when $m$ increases and reduced when $n$ increases. In the middle of $\mathrm{BaTiO}_{3}$ the opposite behavior is observed, with all the amplitudes of the Born-effective charges smaller than in the $\mathrm{BaTiO}_{3}$ bulk, and a trend to reach the bulk values when $m$ increases while they are reduced when $n$ increases. These global evolutions of the Born-effective charges with $n$ and $m$ in the superlattices can be related to the natural disposition to recover the $\mathrm{BaO}$ or $\mathrm{BaTiO}_{3}$ bulk values when, respectively, $n$ or $m$ increases. The evolution of the Born-effective charges of $\mathrm{O}_{1}, \mathrm{Ti}$, and $\mathrm{O}_{2 / 3}$ atoms at the interface are comparable to those in the middle of the $\mathrm{BaTiO}_{3}$ layer but with larger deviations from the bulk amplitudes. The Born-effective charges of the $\mathrm{Ba}$ atom at the interface increases with $m$ and decreases with $n$ but reach values for large $m$ higher than the $\mathrm{BaO}$ or the $\mathrm{BaTiO}_{3}$ bulks. The main conclusion that can be drawn from this discussion is that the Born-effective charges of the atoms in the superlattice are significantly different than those at the bulk level, mainly for the smallest periodicities $m / n$.

Since the Born-effective charges of the atoms are modified, we can expect a modification in the mode polarity of the 
TABLE V. $z z$ components of the Born-effective charges $(e)$ of atoms in the reference paraelectric $\mathrm{BaTiO}_{3} / \mathrm{BaO}$ superlattices with different periodicities $\mathrm{m} / \mathrm{n}$. The first two lines refer the $\mathrm{Ba}$ and $\mathrm{O}$ atoms in the middle of the $\mathrm{BaO}$ layer. Lines third to sixth refer to the $\mathrm{Ba}$, $\mathrm{Ti}$, and $\mathrm{O}$ atoms at the interface. Lines seventh to tenth refer to the $\mathrm{Ba}$, Ti, and $\mathrm{O}$ atoms in the middle of the $\mathrm{BaTiO}_{3}$ layer. $\mathrm{O}_{1}$ refers to the $\mathrm{O}$ in the $\mathrm{BaO}$ plane while $\mathrm{O}_{2 / 3}$ stands for the two equivalent oxygen in the $\mathrm{TiO}_{2}$ planes.

\begin{tabular}{|c|c|c|c|c|c|c|c|c|c|}
\hline & $2 / 2$ & $4 / 2$ & $6 / 2$ & $8 / 2$ & $2 / 4$ & $4 / 4$ & $6 / 4$ & $8 / 4$ & Bulk \\
\hline $\mathrm{Ba}$ & 3.72 & 4.01 & 4.16 & 4.25 & 3.14 & 3.38 & 3.51 & 3.61 & 2.78 \\
\hline $\mathrm{O}$ & -3.10 & -3.34 & -3.46 & -3.53 & -3.15 & -3.38 & -3.53 & -3.63 & -2.78 \\
\hline $\mathrm{Ba}$ & 2.65 & 2.84 & 2.95 & 3.02 & 2.50 & 2.69 & 2.79 & 2.87 & \\
\hline $\mathrm{O}_{1}$ & -3.75 & -4.00 & -4.17 & -4.26 & -3.53 & -3.78 & -3.95 & -4.06 & \\
\hline $\mathrm{Ti}$ & 5.11 & 5.11 & 5.73 & 5.85 & 4.83 & 5.20 & 5.43 & 5.58 & \\
\hline $\mathrm{O}_{2 / 3}$ & -1.68 & -1.82 & -1.88 & -1.92 & -1.59 & -1.71 & -1.78 & -1.83 & \\
\hline $\mathrm{Ba}$ & 2.32 & 2.41 & 2.50 & 2.55 & 2.20 & 2.28 & 2.38 & 2.44 & 2.77 \\
\hline $\mathrm{O}_{1}$ & -4.27 & -4.59 & -4.78 & -4.88 & -4.04 & -4.37 & -4.55 & -4.67 & -5.29 \\
\hline $\mathrm{Ti}$ & & 5.88 & 6.13 & 6.27 & & 5.56 & 5.83 & 6.00 & 6.80 \\
\hline $\mathrm{O}_{2 / 3}$ & & -1.86 & -1.93 & -1.97 & & -1.76 & -1.83 & -1.88 & -2.14 \\
\hline
\end{tabular}

superlattice $\mathrm{FE}_{z}$ instability with respect to the bulk. To compare the evolution of the ferroelectric mode for different periodicities $m / n$, we report in the Fig. 3(a) the dependence with respect the number of $\mathrm{BaTiO}_{3}$ unit cells of the ratio between the oscillator strength and the volume of the unit cell $\left(S / \Omega_{0}\right)$ for $n=2$ and $n=4$. The oscillator strength of one mode is defined as ${ }^{31}$

$$
S_{\alpha \beta}=\sum_{\kappa \alpha^{\prime}} Z_{\kappa, \alpha \alpha^{\prime}}^{*}\left(\eta_{\kappa \alpha^{\prime}}\right)^{*} \sum_{\kappa^{\prime} \beta^{\prime}} Z_{\kappa^{\prime}, \beta \beta^{\prime}}^{*} \eta_{\kappa^{\prime} \beta^{\prime}},
$$

where $Z_{\kappa}^{*}$ are the individual atomic Born-effective charge tensors and $\eta$ is the phonon eigendisplacement vector.

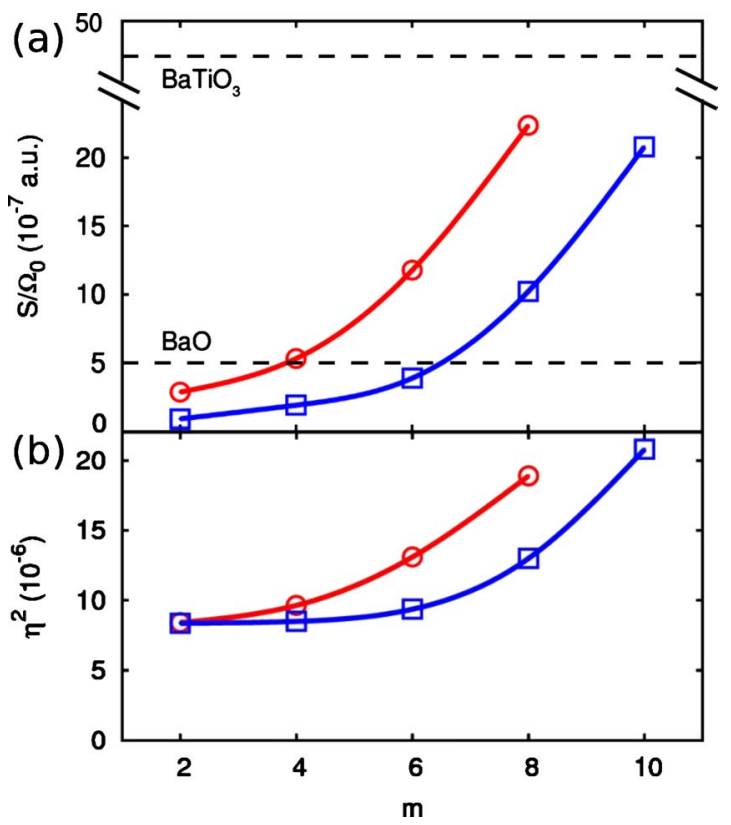

FIG. 3. (Color online) (a) Evolution of the $S / \Omega_{0}$ ratio and (b) $\eta^{2}=\Sigma_{\kappa} \eta_{\kappa}^{*} \eta_{\kappa}$ of the ferroelectric soft mode for $n=2$ (red, circles) and $n=4$ (blue, squares) as a function of the $\mathrm{BaTiO}_{3}$ thickness $m$. The horizontal dashed lines correspond to the $\mathrm{BaTiO}_{3}$ and $\mathrm{BaO}$ bulk limit $S / \Omega_{0}$ values which are, respectively, equal to $43 \times 10^{-7}$ and $5 \times 10^{-7}$ a.u.
This $S / \Omega_{0}$ ratio increases strongly with $m$, evolving rapidly from values smaller than in bulk $\mathrm{BaO}$ at $m=2$ to larger values. At the considered thicknesses, $S / \Omega_{0}$ is much smaller than for bulk $\mathrm{BaTiO}_{3}$ but we can expect that it will reach the $\mathrm{BaTiO}_{3}$ bulk value at large enough $m$. It is clear here that the modification of the $S / \Omega_{0}$ ratio comes either from a modification of the Born-effective charge and/or from a modification of the eigenvector $\eta$. As shown in Table V, the Borneffective charges only tends to increase smoothly with $m$ and cannot be responsible to the strong modification of $S / \Omega_{0}$ shown in Fig. 3(a).

In Fig. 3(b) we report the evolution of the square of the norm of the phonon eigendisplacement vector $\eta^{2}$ with respect to $m$. Here, $\eta^{2}$ increases strongly with $m$, which can be related to a strong enhancement of the ferroelectric eigenvector $\eta$ with the $\mathrm{BaTiO}_{3}$ thickness. Therefore, according to Eq. (15), the evolution of the $S / \Omega_{0}$ ratio can be mainly attributed to the modification of the ferroelectric eigenvector when $m$ is modified.

In summary, this analysis points out that the simple electrostatic model based on the bulk quantities can fail to reproduce the first-principles results because of a strong modification of the ferroelectric eigenvector in the superlattice for the smallest periodicities. This ferroelectric mode is strongly sensitive to the thickness of the $\mathrm{BaTiO}_{3}$ layer in the superlattice and, surprisingly does not reach the $\mathrm{BaTiO}_{3}$ bulk ferroelectric soft mode, even for the largest $m / n$ considered. Compared to other previously studied superlattices such as $\mathrm{SrTiO}_{3} / \mathrm{BaTiO}_{3}$, the modification of the soft-mode pattern could be amplified here due to the fact that the $\mathrm{BaTiO}_{3}$ and the $\mathrm{BaO}$ layer are not sharing the same perovskite structure. This teaches us that the quantitative agreement of the model for $m=2$ could be at least partly fortuitous and that model prediction have to be considered with caution.

\section{Phonon-dispersion curves}

The phonon-dispersion curves between $\Gamma(0,0,0), \mathrm{X}$ $\left(\frac{1}{2}, 0,0\right)$, and $\mathrm{M}\left(\frac{1}{2}, \frac{1}{2}, 0\right)$ points were also calculated, and the results are shown in Fig. 4 for periodicities 2/2, 4/2, and 6/2. 

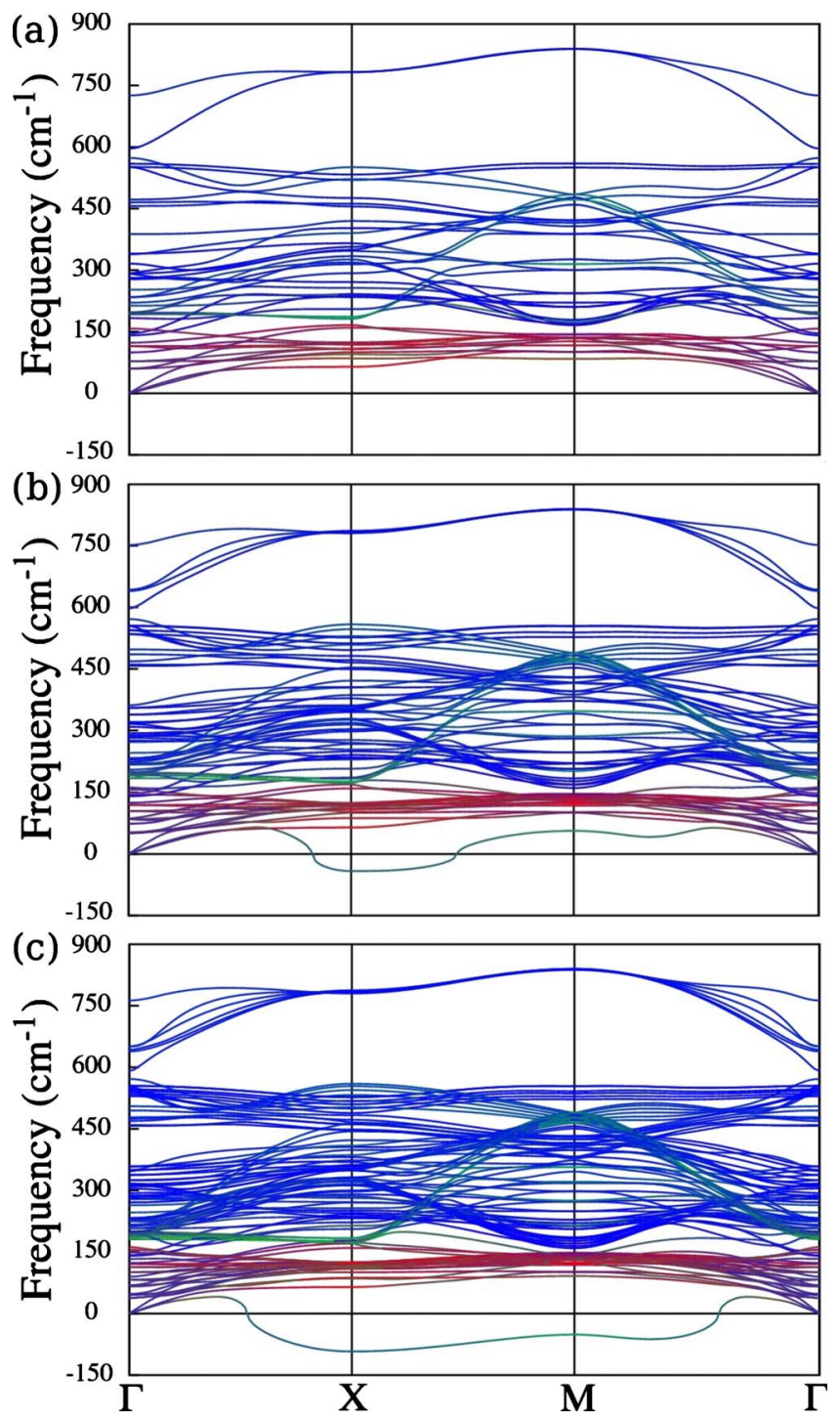

FIG. 4. (Color online) (Color on line) Phonon-dispersion curves for (a) $m / n=2 / 2$, (b) $4 / 2$, and (c) $6 / 2$. The color of the different branches are assigned according to the contribution of each chemical species to the dynamical matrix eigenvector (red for the $\mathrm{Ba}$ atom, green for the $\mathrm{Ti}$ atom, and blue for the $\mathrm{O}$ atom).

At the smallest thickness (2/2), no instability is present, neither at $\Gamma, \mathrm{X}$ nor $\mathrm{M}$ point. This confirms that the ground state of the $2 / 2$ multilayer is the nonpolar $P 4 / \mathrm{mmm}$ phase. However, for the $4 / 2$ periodicity an instability appears at the $X$ zone-boundary point with a frequency of $42 i \mathrm{~cm}^{-1}$, and its magnitude is amplified for larger $m$, with a calculated frequency of $92 i \mathrm{~cm}^{-1}$ for $6 / 2$. Moreover, in Fig. 4(b) a lowfrequency mode is also observed at the $\mathrm{M}$ zone-boundary point for the $4 / 2$ thickness. This mode becomes unstable for the $6 / 2$ periodicity but with an amplitude smaller than the instability at the $\mathrm{X}$ point. The branch responsible for the $\mathrm{M}$ point instability is the same than the one responsible for $\mathrm{X}$ point instability and its dispersion is flat between the $\mathrm{X}$ and $\mathrm{M}$ points while it shifts rapidly to a positive frequency when going from $\mathrm{X}$ or $\mathrm{M}$ point to the $\Gamma$ point. Similar behaviors are also observed for the $m / 4$ periodicities. An X point instability is also observed for $m \geq 4$ and its amplitude is also (a)

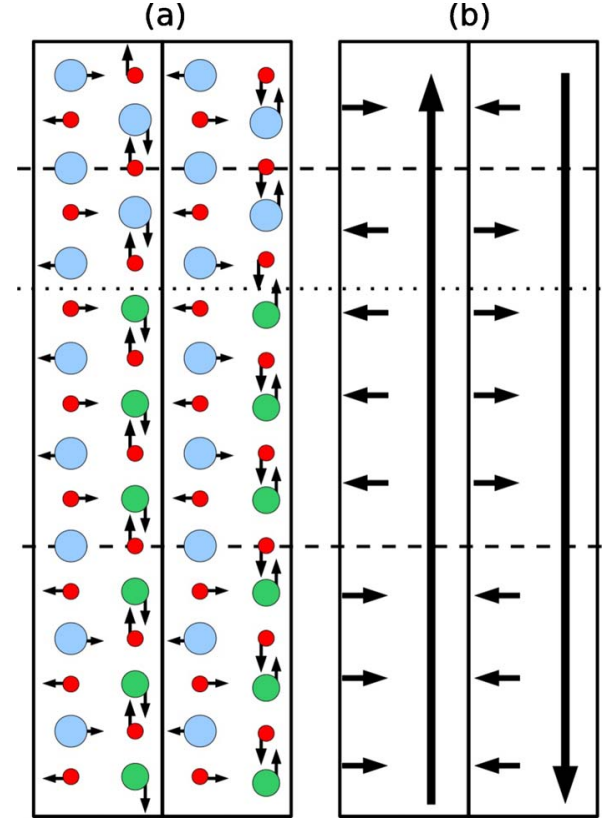

FIG. 5. (Color online) (a) Atomic displacements of the $\mathrm{X}$ point unstable mode for the $6 / 4$ thickness. Two consecutive cells along the $[100]$ direction are shown. Atoms are represented by balls ( $\mathrm{O}$ in red, Ti in green, and $\mathrm{Ba}$ in blue). Dashed lines represent the position of the mirror-symmetry planes of the paraelectric reference structure. Dotted lines are the position of the interfaces. (b) Schematic representation of the direction of polarization resulting from local polar distortions.

amplified with $m$, with essentially the same imaginary frequencies as in the $n=2$ case $\left(42 i \mathrm{~cm}^{-1}\right.$ for $4 / 4$ and $91 i \mathrm{~cm}^{-1}$ for 6/4). In conclusion, for both thicknesses of the $\mathrm{BaO}$ layer, zone-boundary instabilities appear before the zone-center ferroelectric becomes soft.

To clarify the atomic motions related to the $\mathrm{X}$ point instability, we display a schematic picture of the eigendisplacement pattern in Fig. 5 for the $6 / 4$ periodicity. Since the instability is located at the $\mathrm{X}$ point, atoms in consecutive cells along the [100] direction move out of phase. As we can see on Fig. 5, these eigendisplacements can be decomposed into two components: (i) polar distortions along the $z$ direction, only for the atoms along the Ti-O chains, and (ii) in-plane motions, only for $\mathrm{Ba}$ and $\mathrm{O}$ atoms which are along the $\mathrm{Ba}-\mathrm{O}$ chains parallel to the Ti-O chains. The amplitudes of these in-plane displacements are however much smaller than the out-of-plane displacements, meaning that the Ti-O distortions along the $z$ direction dominate the total motions (bluegreen color of the unstable branch at the X point in Fig. 4). Moreover, on the two $\mathrm{BaO}$ atomic planes corresponding to the two mirror planes of symmetry in the $z$ direction, the in-plane motions completely disappear. Additionally, we can notice that the polar distortions along the $z$ direction remain in the $\mathrm{BaO}$ layer but only for the atoms aligned with the Ti-O chain, in order to preserve the flow of large polarization currents along those chains.

Since in consecutive unit cells along the [100] direction the polar displacements are in opposite directions, these $\mathrm{X}$ point instabilities are related to an antiferroelectric distor- 
tion. To highlight these issues, we report also in Fig. 5(b) schematic vectors showing the direction of main local dipolar moments. From this picture, it is clear that along the $z$ directions we have antiferroelectric distortions, with alternating chains of up and down polarization. For the in-plane $\mathrm{Ba}$ and $\mathrm{O}$ motions, the atoms move only in the [100] direction, giving rise to dipolar moments oriented along the $x$ direction. Between the two mirror-symmetry planes, these in-plane dipolar moments keep the same orientation, but it is inverted after crossing these symmetry planes. Interestingly, this inversion does not take place at the interface of the supercell but in the middle of each layer.

\section{DISCUSSION}

The thickness evolution of the phonon-dispersion curves of the $\mathrm{BaTiO}_{3} / \mathrm{BaO}$ superlattice, as discussed in Sec. IV C, can be understood in relationship with those of bulk $\mathrm{BaTiO}_{3}$. At the bulk level, $\mathrm{BaTiO}_{3}$ was shown to exhibit a chainlike ferroelectric instability in real space, with weak interchain interactions, yielding ferroelectric modes similarly unstable at $\Gamma, X$, and $M$ points. ${ }^{39,40}$ The ferroelectric mode at $\Gamma$ is highly polar. In the superlattice, due to the difficulty to polarize $\mathrm{BaO}$, it will be associated to a huge depolarizing field which will strongly harden it. At the opposite, the zoneboundary ferroelectric modes at $\mathrm{X}$ and $\mathrm{M}$ are globally nonpolar. They will therefore not induce any macroscopic depolarizing field and will so keep a much stronger tendency to be unstable than the $\Gamma$ mode. Consequently, the zoneboundary modes will always be more unstable than the $\Gamma$ mode in the superlattice, yielding an antiferroelectric ground state. It is only in the limit of thick $\mathrm{BaTiO}_{3}$ layers, for which the depolarizing field tends to vanish, that we will recover the usual ferroelectric ground state. It is also worth to notice that, for $n=2$ and 4, the instability at X similarly appears at $m=4$, which corresponds to what was previously reported as the approximative length of the correlation volume $(\mathrm{m}$ $\approx 4-5$ ) required to induce a ferroelectric instability. ${ }^{39,41}$

The evolution of the ground state of the superlattice with the thicknesses of the layers can therefore be summarized as follows: (i) for the smallest $\mathrm{BaTiO}_{3}$ thicknesses $m<4$, the system does not exhibit any ferroelectric instability and the supercell remains paraelectric. (ii) For larger $\mathrm{BaTiO}_{3}$ thicknesses $(m>4)$ but small ratio $m / n$, the ferroelectric instability at $\Gamma$ is suppressed by the effect of the depolarizing field; there is only an instability at $\mathrm{X}$ and the system is antiferroelectric. (iii) As the ratio $m / n$ increases, the ferroelectricity will progressively expand from $X$ to $\Gamma$ and we can expect the formation of ferroelectric domains of increasing sizes. (iv) Only when $m \gg n$, the superlattice will tend to the purely ferroelectric ground state.

Antiferroelectricity was also previously reported in some ferroelectric/incipient-ferroelectric and incipient- ferroelectric/paraelectric bicolor superlattices in which the two layers are made of perovskite compounds. ${ }^{18,22}$ In Ref. 18 it was suggested that the origin of antiferroelectricity in such system could be correlated with the imposition of a $B$-site ordering. More generally, we propose here that the appearance of an antiferroelectric ground state in superlattices including a less polarizable layer is rather related to the depolarizing fields which penalize the FE instability with respect to the antiferroelectric instability.

We notice that the $\mathrm{BaTiO}_{3} / \mathrm{BaO}$ superlattices present similarities with Ruddlesden-Popper $A_{n+1} B_{n} O_{3 n+1}$ materials which can be viewed as the stacking of alternating perovskite and rocksalt layers. ${ }^{26}$ In the latter, however, there is no continuity of the Ti-O chains from one perovskite block to the next one. This will prevent the possibility of huge polarization currents along the stacking direction as those associated to the ferroelectric mode in $\mathrm{BaTiO}_{3} / \mathrm{BaO}$ system and could explain why Ruddlesden-Popper materials do not develop any tendency to become ferroelectric with polarization aligned along the stacking direction. ${ }^{27}$

\section{CONCLUSIONS}

In this paper we have studied, from first-principles, superlattices combining $m$ unit cells of $\mathrm{BaTiO}_{3}$ with $n$ unit cells of $\mathrm{BaO}$. Although $\mathrm{BaTiO}_{3}$ is ferroelectric at the bulk level, we have shown that the superlattice can present an antiferroelectric ground state and explained that by the hardening of the ferroelectric mode with respect to the antiferroelectric mode due to depolarizing field issues.

This behavior is quite general. While epitaxial strain was already previously reported to be able to modify the competition between ferroelectric and antiferrodistortive instabilities, we propose that playing with the depolarizing field in ferroelectric/insulator superlattices is another practical way to tune the competition between polar and nonpolar instabilities. We have demonstrated here the possibility to engineer an antiferroelectric ground state in a system combining ferroelectric and antiferroelectric instabilities. We anticipate that in systems with competing ferroelectric and antiferrodistortive instabilities, it could similarly favor an antiferrodistortive ground state. We hope that these results will motivate further studies of ferroelectric/insulator superlattices.

\section{ACKNOWLEDGMENTS}

This work was supported by the European Project No. CP-FP 228989-2 OxIDes of the Seventh Framework Program, the European Multifunctional Institute, and the Interuniversity Attraction Poles Program (P6/42)_Belgian State-Belgian Science Policy. J.J. acknowledges financial support of the Spanish Ministry of Science and Innovation through the MICINN under Grant No. FIS2009-12721-C0402. E.B. also acknowledges FRS-FNRS Belgium. 
${ }^{1} \mathrm{Ph}$. Ghosez and J. Junquera, Handbook of Theoretical and Computational Nanotechnology (ASP, Stevenson Ranch, 2006), Vol. 9, p. 623.

${ }^{2}$ M. Dawber, K. M. Rabe, and J. F. Scott, Rev. Mod. Phys. 77, 1083 (2005).

${ }^{3}$ J. Junquera and Ph. Ghosez, Nature (London) 422, 506 (2003).

${ }^{4}$ M. Stengel and N. Spaldin, Nature (London) 443, 679 (2006).

${ }^{5}$ P. Aguado-Puente and J. Junquera, Phys. Rev. Lett. 100, 177601 (2008)

${ }^{6}$ M. Stengel, D. Vanderbilt, and N. Spaldin, Nature Mater. 8, 392 (2009).

${ }^{7}$ M. Stengel, D. Vanderbilt, and N. A. Spaldin, Phys. Rev. B 80, 224110 (2009).

${ }^{8}$ B. D. Qu, W. L. Zhong, and R. H. Prince, Phys. Rev. B 55, 11218 (1997).

${ }^{9}$ A. M. George, J. Íñiguez, and L. Bellaiche, Nature (London) 413, 54 (2001).

${ }^{10}$ N. Huang, Z. Liu, Z. Wu, J. Wu, W. Duan, B.-L. Gu, and X.-W. Zhang, Phys. Rev. Lett. 91, 067602 (2003).

${ }^{11}$ J. B. Neaton and K. M. Rabe, Appl. Phys. Lett. 82, 1586 (2003).

${ }^{12}$ K. Johnston, X. Huang, J. B. Neaton, and K. M. Rabe, Phys. Rev. B 71, 100103 (2005).

${ }^{13}$ M. Dawber, C. Lichtensteiger, M. Cantoni, M. Veithen, $\mathrm{Ph}$. Ghosez, K. Johnston, K. M. Rabe, and J.-M. Triscone, Phys. Rev. Lett. 95, 177601 (2005).

${ }^{14}$ E. Bousquet, M. Dawber, N. Stucki, C. Lichtensteiger, P. Hermet, S. Gariglio, J.-M. Triscone, and Ph. Ghosez, Nature (London) 452, 732 (2008).

${ }^{15}$ M. Sepliarsky, S. R. Phillpot, D. Wolf, M. G. Stachiotti, and R. L. Migoni, Phys. Rev. B 64, 060101 (2001).

${ }^{16}$ T. Shimuta, O. Nakagawara, T. Makino, S. Arai, H. Tabata, and T. Kawai, J. Appl. Phys. 91, 2290 (2002).

${ }^{17}$ E. D. Specht, H.-M. Christen, D. P. Norton, and L. A. Boatner, Phys. Rev. Lett. 80, 4317 (1998).

${ }^{18}$ J. Sigman, D. P. Norton, H. M. Christen, P. H. Fleming, and L. A. Boatner, Phys. Rev. Lett. 88, 097601 (2002).

${ }^{19}$ V. A. Stephanovich, I. A. Luk'yanchuk, and M. G. Karkut, Phys. Rev. Lett. 94, 047601 (2005).

${ }^{20}$ F. A. Urtiev, V. G. Kukhar, and N. A. Pertsev, Appl. Phys. Lett. 90, 252910 (2007).

${ }^{21}$ H. N. Lee, H. N. Christen, M. F. Chrisholm, C. M. Rouleau, and D. H. Lowndes, Nature (London) 433, 395 (2005).

${ }^{22}$ H. M. Christen, E. D. Specht, S. S. Silliman, and K. S. Harsha- vardhan, Phys. Rev. B 68, 020101 (2003).

${ }^{23}$ T. Tsurumi, T. Harigai, D. Tanaka, S.-M. Nam, H. Kakemoto, S. Wada, and K. Saito, Appl. Phys. Lett. 85, 5016 (2004).

${ }^{24}$ R. A. McKee, F. J. Walker, and M. F. Chisholm, Phys. Rev. Lett. 81, 3014 (1998).

${ }^{25}$ J. Junquera, M. Zimmer, P. Ordejón, and Ph. Ghosez, Phys. Rev. B 67, 155327 (2003).

${ }^{26}$ S. N. Ruddlesden and P. Popper, Acta Crystallogr. 10, 538 (1957).

${ }^{27}$ S. M. Nakhmanson, Phys. Rev. B 78, 064107 (2008).

${ }^{28}$ S. M. Nakhmanson and I. Naumov, Phys. Rev. Lett. 104, 097601 (2010)

${ }^{29}$ X. Gonze, J.-M. Beuken, R. Caracas, F. Detraux, M. Fuchs, G.-M. Rignanese, L. Sindic, M. Verstraete, G. Zerah, F. Jollet, M. Torrent, A. Roy, M. Mikami, Ph. Ghosez, J.-Y. Raty, and D. Allan, Comput. Mater. Sci. 25, 478 (2002).

${ }^{30}$ M. Teter, Phys. Rev. B 48, 5031 (1993).

${ }^{31}$ X. Gonze and C. Lee, Phys. Rev. B 55, 10355 (1997).

${ }^{32}$ R. A. McKee, F. J. Walker, J. R. Conner, E. D. Speccht, and D. E. Zelmon, Appl. Phys. Lett. 59, 782 (1991).

${ }^{33}$ R. A. McKee, F. J. Walker, and M. F. Chisholm, Science 293, 468 (2001).

${ }^{34}$ Experimentally, the lattice constants of $\mathrm{BaO}$ and $\mathrm{SrTiO}_{3}$ fit nearly exactly. The small epitaxial strain of $0.5 \%$ that we report, together with the underestimate of $\omega_{\mathrm{TO}}$ (and the related overestimate of the dielectric constant), is an artifact of the LDA but $\mathrm{BaO}$ remains properly described as a paraelectric so that it does not affect our main conclusions.

${ }^{35}$ E. Bousquet, N. A. Spaldin, and Ph. Ghosez, Phys. Rev. Lett. 104, 037601 (2010).

${ }^{36}$ O. Diéguez, K. M. Rabe, and D. Vanderbilt, Phys. Rev. B 72, 144101 (2005).

${ }^{37}$ N. Sai, K. M. Rabe, and D. Vanderbilt, Phys. Rev. B 66, 104108 (2002).

${ }^{38}$ We checked that taking into account the relaxation of $c$ with respect to the polarization does not affect the model predictions.

${ }^{39} \mathrm{Ph}$. Ghosez, J.-P. Michenaud, and X. Gonze, Phys. Rev. B 58, 6224 (1998).

${ }^{40} \mathrm{Ph}$. Ghosez, E. Cockayne, U. V. Waghmare, and K. M. Rabe, Phys. Rev. B 60, 836 (1999).

${ }^{41}$ G. Geneste, E. Bousquet, and Ph. Ghosez, J. Comput. Theor. Nanosci. 5, 517 (2008). 